\title{
La didáctica universitaria y el rendimiento académico en una Universidad de Lima
}

\author{
University teaching and academic performance in a University of Lima
}

Dante O. Neyra ${ }^{1}$.

RESUMEN

Objetivo: Determinar la relación entre la didáctica del docente y el rendimiento académico de los estudiantes de la Escuela Académica Profesional de Estadística de la Facultad de Ciencias Matemáticas de la Universidad Nacional Mayor de San Marcos (Semestre 2015-II). Materiales y Métodos: El tipo de estudio fue básico, descriptivo-correlacional, el diseño de investigación fue no experimental de corte transversal, la población bajo estudio fue de 225 estudiantes de la especialidad de Estadística matriculados en el Semestre 2015-II, donde la muestra fue tomada de forma probabilística a través del muestreo aleatorio simple, se diseñó y aplicó un cuestionario a los estudiantes a fin de recoger datos de las variables bajo estudio, el método de investigación fue el hipotético-deductivo, se realizó la validez de constructo a través del análisis factorial, el análisis de confiabilidad se realizó a través del modelo de consistencia interna (alfa de Cronbach) para cada dimensión, el cual dio una buena fiabilidad. Para el análisis de datos se utilizó el análisis de correspondencias. Resultados: Se evidenció una relación significativa entre la didáctica universitaria y el rendimiento académico $\left(X^{2}=13.849\right.$; p-valor $\left.=0.008\right)$; así también, los estudiantes de Estadística poseen un rendimiento académico regular (50\%). Conclusión: Existe una asociación entre la didáctica universitaria y el rendimiento académico, y los estudiantes poseen un rendimiento regular.

Palabras clave: didáctica, didáctica universitaria, rendimiento académico.

\begin{abstract}
Objective: To determine the relationship between university teaching and academic performance of students in the academic professional school for Statistics of Faculty of Mathematics - San Marcos University (Period 2015-II). Materials and Methods: It was a basic study, descriptive-correlational, the research design was non-experimental cross-section, the population under study were 225 undergraduate students enrolled in the School of Statistics registered in Period 2015-II, where the sample was taken in a probabilistic form, through simple random sampling. An instrument was designed and applied specifically for this study, the research method was the deductive-hypothetic method. About the construct validity, it was realized applied the factorial analysis, and the reliability analysis was realized applying the Cronbach's alpha by dimensions. To data analysis it was used the correlation analysis. Results: There was a significant relationship between university teaching and academic performance of students $\left(X^{2}=13.849\right.$; $\mathrm{p}$-valor $\left.=0.008\right)$. Academic performance of students was regular (50\%). Conclusion: There is an association between university teaching and academic performance and the academic performance of students is regular.
\end{abstract}

Key words: teaching, university teaching, academic performance.

\section{INTRODUCCIÓN}

La didáctica es una de las principales preocupaciones de la pedagogía, dada su trascendencia en el proceso enseñanzaaprendizaje. Antiguamente, se esperaba encontrar el método didáctico con el cual enseñar todo a todas las personas (Navarro,
2009, 2011), tal como lo deja ver Comenio (1998) en su libro Didáctica magna (publicado por primera vez en 1630), cuando manifiesta que "[...] con un solo y mismo método [didáctico] se puede instruir y formar una juventud de índole tan diversa [...]" (p. 34).

${ }^{1}$ Estadístico y Educador por la Universidad Nacional Mayor de San Marcos. Lima. Perú. 
A decir de Schmieder y Schmieder (1963), la didáctica es una ciencia dado que lleva en sí sus propias leyes o principios, los cuales obtiene de la misión que ha de realizar. En cuanto a los modelos pedagógicos contemporáneos, la escuela se mueve en un contexto social, histórico y político con el que, necesariamente, busca una coherencia. En ese sentido, la didáctica no es neutral; por el contrario, es el nivel más operativo de un entramado de teorías que se ocupa del estudio de los procesos, sujetos y elementos de la educación formal (Navarro, 2009, 2011).

Se puede definir la didáctica como el estudio del proceso de enseñanza-aprendizaje, proceso que se da en interacción constante entre los actores involucrados como son el docente y el discente, sobre el tema a ser aprendido, en un contexto determinado, con métodos de enseñanza-aprendizaje y formas de evaluación. Bajo esa definición se circunscribe la didáctica universitaria, es decir, es el estudio del proceso de enseñanza-aprendizaje que se manifiesta en entornos universitarios, es decir, la didáctica universitaria se puede considerar como una didáctica diferencial comprometida con lo significativo de los aprendizajes del futuro profesional, con su desarrollo personal y con el potencial de su inteligencia en función de las exigencias del contexto socio-político (Mallart, 2001).

Así también, el rendimiento académico en el entorno universitario es un factor importante en el contexto de la calidad de la educación superior, dado que es un indicador que permite una aproximación a la realidad educativa universitaria (Díaz, Peio, Arias, Escudero, Rodríguez, Vidal, 2002, citado por Garbanzo, 2007). En ese sentido, el rendimiento académico se define como el indicador de las capacidades que el discente ha adquirido como consecuencia de un proceso de formación. A decir de Touron (1984), el rendimiento académico se expresa en una calificación cuantitativa en términos vigesimales, $\mathrm{y}$ cualitativo en bajo, regular y alto, el cual reflejará el resultado de los objetivos preestablecidos.

Desde la perspectiva del docente universitario, el concepto de la didáctica universitaria comienza a tomar importancia; sin embargo, en entornos de las especialidades de ciencias e ingenierías, dicha importancia aún no es significativa. En particular, en el entorno social de estudio, en la Escuela Académico-Profesional de Estadística de la Universidad Nacional Mayor de San Marcos
(UNMSM), la práctica docente usualmente no considera la didáctica como una forma de mejorar los aprendizajes en el estudiante $\mathrm{y}$, por tanto, su rendimiento académico.

La presente investigación se enfoca en dar una mirada general acerca de la didáctica en un entorno universitario, y si esta didáctica universitaria se relaciona al rendimiento académico de los estudiantes, de manera significativa.

\section{MATERIALES Y METODOS}

La investigación fue de tipo básica, descriptiva-correlacional, no experimental de corte transversal. La población fueron todos los estudiantes matriculados en la EAP de Estadística de la UNMSM en el Semestre 2015-II. El diseño muestral fue unietápico, el tipo de muestreo fue el muestreo aleatorio simple (MAS), obteniéndose un tamaño de muestra de 80 estudiantes, y la selección se realizó de forma sistemática. Se aplicó un cuestionario, elaborado en la investigación, a los estudiantes seleccionados. Previamente, se realizó la validez a través del análisis factorial $(\mathrm{KMO}=0,794)$, y para la confiabilidad se aplicó el modelo de consistencia interna de Cronbach, el cual utiliza el coeficiente Alfa de Cronbach. Los coeficientes dieron una buena fiabilidad en cada una de las dimensiones de la didáctica universitaria. En los resultados se aplicó análisis descriptivo y el análisis de correspondencias para la comprobación de la hipótesis de investigación.

\section{RESULTADOS}

A continuación se presentan los análisis descriptivos más relevantes para luego dar paso al análisis respecto a la comprobación de la hipótesis.

Tabla 1. Análisis descriptivo del rendimiento académico.

\begin{tabular}{lcc}
\hline & Frecuencia & Porcentaje \\
\hline Bajo & 21 & $26,2 \%$ \\
Regular & 40 & $50,0 \%$ \\
Alto & 19 & $23,8 \%$ \\
Total & 80 & $100,0 \%$ \\
\hline
\end{tabular}

En cuanto al rendimiento académico, el $50 \%$ de los estudiantes tuvieron un rendimiento académico regular, el $26,2 \%$ de 
La didáctica universitaria y el rendimiento académico en una Universidad de Lima Neyra DO los estudiantes tuvo un rendimiento académico bajo, y el 23,8\% de los estudiantes tuvieron un rendimiento académico alto (Tabla 1).

Tabla 2. Análisis descriptivo de la didáctica universitaria.

\begin{tabular}{lcc}
\hline & Frecuencia & Porcentaje \\
\hline Pésima & 8 & $10,0 \%$ \\
Regular & 66 & $82,5 \%$ \\
Buena & 6 & $7,5 \%$ \\
Total & 80 & $100,0 \%$ \\
\hline
\end{tabular}

Respecto a la didáctica universitaria, el $82,5 \%$ de los estudiantes indicó que la didáctica universitaria es regular, el $10 \%$ indicó que es pésima y el 7,5\% indicó que la didáctica universitaria es buena (Tabla 2).

El análisis estadístico a través del estadístico $X^{2}$ (Chi-cuadrado) utilizado en el análisis previo de correspondencias, evidencia la existencia de una relación significativa entre la didáctica universitaria y el rendimiento académico, según los estudiantes encuestados (Tabla 3).
Tabla 3. Estadístico de asociación $X^{2}$, entre la didáctica universitaria y el rendimiento académico.

\begin{tabular}{cc}
\hline & Rendimiento académico \\
\cline { 2 - 2 } $\begin{array}{c}\text { Didáctica } \\
\text { universitaria }\end{array}$ & Estadístico $X^{2}=13.849 * *$ \\
\hline$p=0.008 ; p<0.05$ &
\end{tabular}

El mapa perceptual indica la relación significativa entre la didáctica universitaria y el rendimiento académico, toda vez que se observan claramente asociaciones entre los atributos de ambas variables. Así, los estudiantes que opinan que la didáctica universitaria es pésima, tienen un rendimiento académico bajo. Los estudiantes que opinan que la didáctica universitaria es regular, tienen un rendimiento académico regular. Finalmente, los estudiantes que opinan que la didáctica universitaria es buena, tienen un rendimiento académico alto (Fig. 1).

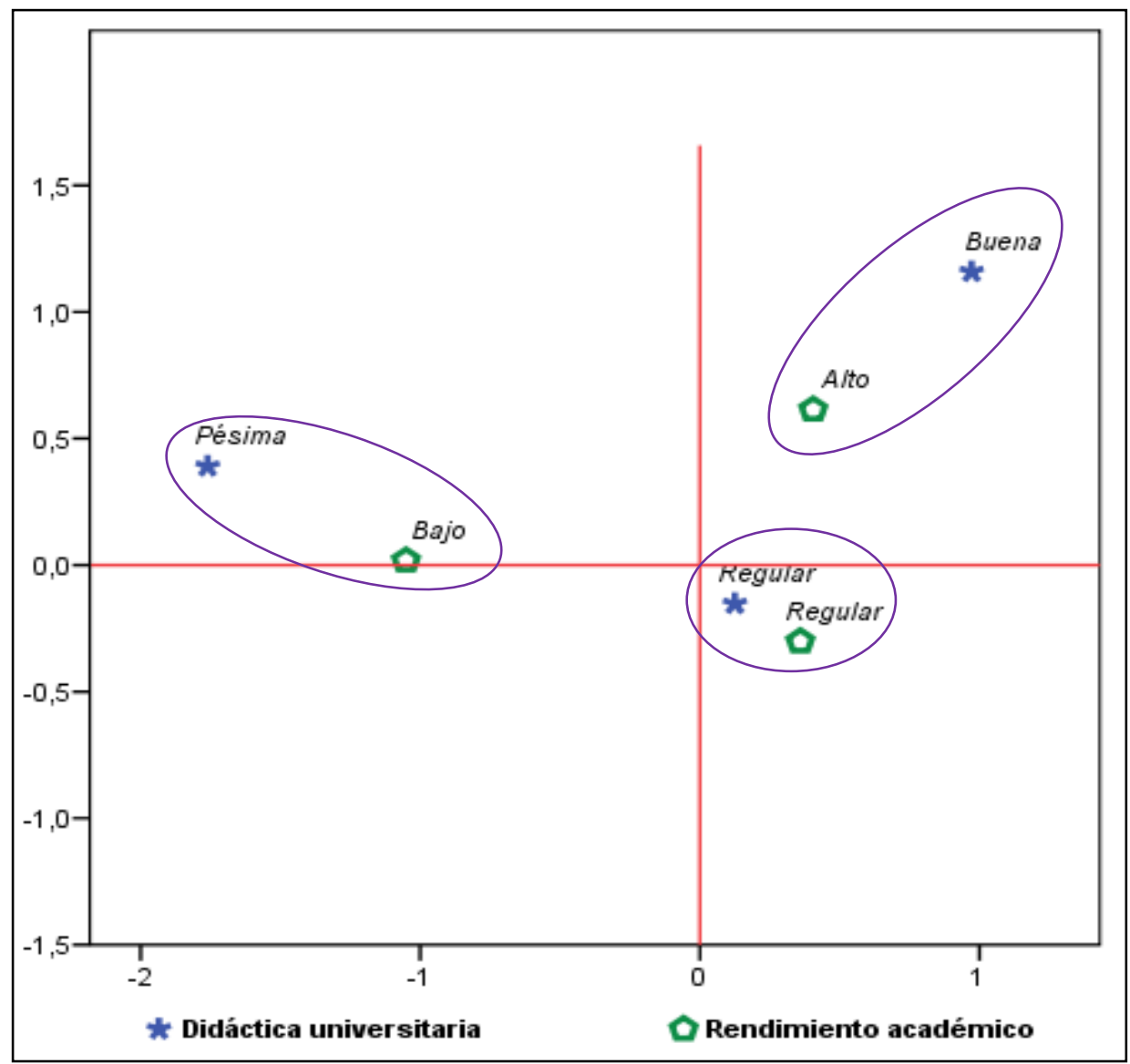

Fig. 1. Mapa perceptual de la asociación entre los atributos de la didáctica universitaria y el rendimiento académico. 


\section{DISCUSIÓN}

Los hallazgos presentados indican una fuerte relación entre la didáctica universitaria y el rendimiento académico. Esto va de acuerdo a lo mostrado por Sánchez (2010) quien manifiesta una relación entre el acto didáctico y el rendimiento académico en contextos educativos superiores. En ese mismo sentido se debe destacar la importancia de las actividades docentes en general, toda vez que éstas influyen en el rendimiento académico de los estudiantes, y en ese sentido, los resultados de la investigación apoyan las conclusiones de González (2004) quien evidencia una influencia entre las actividades docentes y el rendimiento académico. Este hallazgo, confirma, de cierta forma, la importancia de la didáctica en entornos de enseñanza superior (Zabalza, 2005; Rey, 2007; Herrán, 2001), y que es necesario considerar dentro del contexto universitario.

A nivel del rendimiento académico, los resultados evidencian que los estudiantes poseen un promedio ponderado de regular, lo que va de acuerdo a lo mostrado por Moromi (2002), y los resultados de Sánchez (2010) quien evidencia que los estudiantes tienen un rendimiento medio, así como Torres y Merino (2010) quienes muestran similares resultados.

\section{CONCLUSIONES}

Se ha evidenciado la existencia significativa de una asociación entre la didáctica universitaria y el rendimiento académico, en el contexto de la Escuela Académico-Profesional de Estadística de la Facultad de Ciencias Matemáticas de la UNMSM. En ese sentido, la didáctica de los docentes que enseñan en la Escuela de Estadística, está relacionada a los niveles de rendimiento académico de los estudiantes quienes reciben dicha enseñanza. Los resultados también evidencian que los estudiantes de estadística de la Facultad de Ciencias Matemáticas de la UNMSM, poseen un rendimiento académico regular.

\footnotetext{
AUTOR DE CORRESPONDENCIA

Dante O. Neyra Salas, Mg.

Stadisti-K Asociados

e-mail: dneyra@stadisti-k.com
}

\section{REFERENCIAS BIBLIOGRÁFICAS}

1. Comenio, J. (1998). Didáctica Magna (8va Ed.). México: Porrúa.

2. Garbanzo, G. (2007). Factores asociados al rendimiento académico en estudiantes universitarios, una reflexión desde la calidad de la educación superior pública. Revista Educación, 31(1), 43-63.

3. González, I. (2004). Realización de un análisis discriminante explicativo del rendimiento académico en la universidad. Revista de Investigación Educativa, 22(1), 43-59.

4. Herrán, A. (2001). Didáctica Universitaria: La cara dura de la universidad. Revista Tendencias Pedagógicas, 6, 11-38.

5. Mallart, J. (2001). Didáctica: concepto, objeto y finalidades. En N. Rajadell \& F. Sepúlveda (Eds.), Didáctica general para psicopedagogos (pp. 25-60). España: Universidad Nacional de Educación a Distancia-UNED.

6. Moromi, H. (2002). La Influencia de la Ejecución Curricular y el Uso de Medios y materiales en el rendimiento académico de los estudiantes de la facultad de Odontología de la Universidad Nacional Mayor de San Marcos. Recuperado de Cybertesis, UNMSM.

7. Navarro, B. (2011). Didáctica general (Antología comentada). PROTEC. Lima: UNMSM.

8. Navarro, B. (2009). Didáctica general (referentes teóricos). Programa de Complementación Pedagógica. Lima: CEPREDIM, UNMSM.

9. Rey, J, (2007). El Procesamiento Didáctico en los Entornos Virtuales de Aprendizaje. En E. Barrientos (Ed.), Didáctica de la Educación Superior II (pp. 152-156). Lima: UNMSM.

10. Sánchez, T. (2010). Influencia del acto didáctico en el rendimiento de los alumnos del V ciclo del curso de metodología de la investigación en la Facultad de Educación de la Universidad Nacional Mayor de San Marcos. Recuperado de Cybertesis, UNMSM.

11. Schmieder, A. \& Schmieder J. (1963). Didáctica General (7ma Ed.). Buenos Aires: Losada S.A.

12. Torres, M. \& Merino, J. (2010). La ejecución real del Plan Curricular y el uso de los medios y materiales en el mejoramiento del rendimiento académico de los estudiantes de la especialidad 
Administración de la Facultad de Ciencias Administrativas y Turismo de la Universidad Nacional de Educación UNE. Recuperado del Instituto de Investigación, UNE.

13. Touron, J. (1984). Factores del rendimiento académico en la universidad. Pamplona: EUNSA.

14.Zabalza, M. (2005, febrero). Didáctica Universitaria. Conferencia pronunciada en la Pontificia Universidad Javeriana, Cali, Colombia.

Recibido: 05/08/2016

Aceptado: 20/10/2016 